\title{
Salt Stress-Induced Anthocyanin Biosynthesis Genes and MATE Transporter Involved in Anthocyanin Accumulation in Daucus carota Cell Culture
}

\author{
Kirti R. Saad, Gyanendra Kumar, Sandeep N. Mudliar, Parvatam Giridhar, and Nandini P. Shetty*
}

Cite This: ACS Omega 2021, 6, 24502-24514

Read Online

ABSTRACT: Anthocyanins biosynthesis is a well-studied biosynthesis pathway in Daucus carota. However, the scale-up production at the bioreactor level and transporter involved in accumulation is poorly understood. To increase anthocyanin content and elucidate the molecular mechanism involved in accumulation, we examined D. carota cell culture in flask and bioreactor for 18 days under salt stress $\left(20.0 \mathrm{mM} \mathrm{NH} \mathrm{NO}_{3} / 37.6 \mathrm{mM} \mathrm{KNO}_{3}\right)$ at 3 day intervals. The expression of anthocyanin biosynthesis and putative MATE (multidrug and toxic compound extrusion) transporter expression was analyzed by qRT-PCR. It was observed that there was a significant enhancement of anthocyanin in the bioreactor compared to the control culture. A correlation was observed between the expression of MATE and the anthocyanin biosynthesis

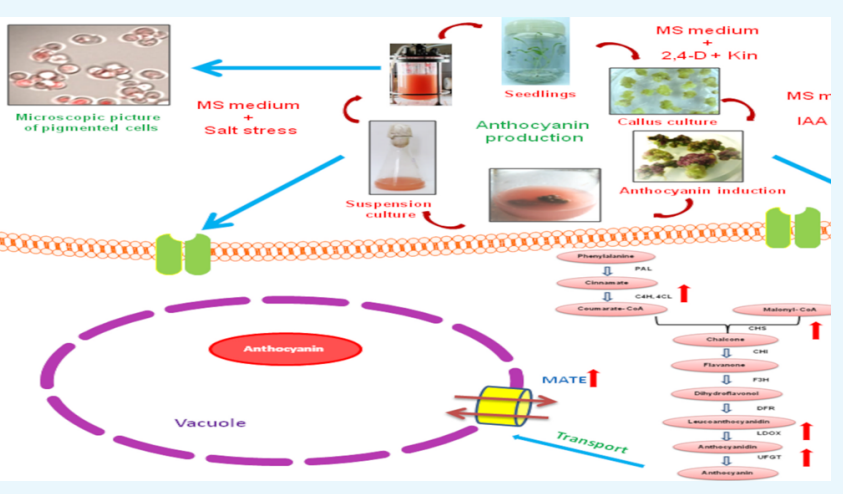
genes (CHS, C4H, LDOX, and UFGT) on the 9th day in a bioreactor, where maximum anthocyanin accumulation and expression was detected. We hypothesize the involvement of MATE in transporting anthocyanin to tonoplast in $D$. carota culture under salt stress.

\section{INTRODUCTION}

The anthocyanins are water-soluble plant pigments that belong to the flavonoid family of polyphenol phytochemicals. They possess anti-cancer, anti-microbial, anti-diabetic, anti-obesity, and anti-inflammatory properties in addition to preventing cardiovascular diseases. ${ }^{1,2}$ The anthocyanin biosynthesis pathway has been well characterized, and it is conserved in many plant species. ${ }^{3}$ The anthocyanin pathway genes are shown in Figure 1, including early genes \{phenylalanine ammonia-lyase $(P A L)$, cinnamate-4-hydroxylase $(\mathrm{C} 4 \mathrm{H})$, 4-coumarate: CoAligase $(C L)$, chalcone synthase $(\mathrm{CHS})$, chalcone isomerase $(\mathrm{CHI})$ \}, and late genes fflavanone 3-hydroxylase $(\mathrm{F} 3 \mathrm{H})$, flavonoid $3^{\prime}$-hydroxylase $\left(F 3^{\prime} H\right)$, dihydroflavonol 4-reductase (DFR), leucoanthocyanidin dioxygenase (LDOX) and UDPglucose: flavonoid 3-O-glucosyltransferase (UFGT) \}.

Anthocyanins are synthesized at the cytosolic surface of the endoplasmic reticulum and then transported into the vacuole for storage. However, the involvement of transporter in the accumulation of anthocyanin remains poorly understood. Many types of mechanisms, including glutathione $S$-transferases (GSTs), multidrug and toxic extrusion (MATE), and ATP-binding cassette $(A B C)$ proteins, are known to be responsible for the transport of anthocyanin in plants. ${ }^{4}$ Different anthocyanin transporters are involved in transporting different anthocyanins from cytoplasm to vacuole in plants. Earlier studies have shown the involvement of MtMATE-2 in
Medicago Truncatula ${ }^{5}$ and AM1 and AM3 in Vitis vinifera. ${ }^{6}$ However, there is less evidence of the MATE transporter involvement in the transport of anthocyanin in the callus culture of Daucus carota. The relationship between the MATE transporter and flavonoid vacuole accumulation has sparked a lot of interest.

In general, an exogenous supply of biotic/abiotic elicitors to various culture systems causes a plant under stress like a cellular environment. Incorporating the elicitors into in vitro culture mimics the culture environment similar to natural conditions under stress related damage in the plant, which enhanced the yield of the target biomolecule. ${ }^{7}$ The development of natural colorants with pharmaceutical properties and health benefits, using plant tissue culture, has given rise to new research interest aimed at producing these valuable phytochemicals efficiently without elicitors to reduce the cost of production. This also provides enhanced control of the chemical and physical production environments. ${ }^{8}$ The plant cell culture technology shows the potential for large-scale

Received: June 5, 2021

Published: September 15, 2021 
<smiles>N[C@@H](Cc1ccccc1)C(=O)O</smiles>

Phenylalanine<smiles>CSC(=O)/C=C/c1ccc(O)cc1</smiles>

p-Coumaroyl-CoA

Malonyl-CoA<smiles>CC(=O)OCC(=O)CC(=O)O</smiles><smiles>C1CCCC1</smiles>
$\mathrm{CHS}$<smiles>O=C(/C=C/c1ccccc1)c1ccccc1</smiles>
Chalcone<smiles>O=C1CC(c2ccc(O)cc2)Oc2cc(O)cc(O)c21</smiles>

Naringenin<smiles></smiles>

Cyanidin<smiles>Oc1ccc2c(c1)OC(c1ccc(O)c(O)c1)C(O)C2O</smiles><smiles>O=c1c(O)c(-c2ccc(O)c(O)c2)oc2cc(O)cc(O)c12</smiles>

F3'H<smiles>CC(C)CC1C(=O)c2c(O)cc(O)cc2OC1c1ccc(O)cc1</smiles><smiles>OCC1OC(Oc2cc(-c3ccc(O)c(O)c3)c(O)cc2O)C(O)C(O)C(O)C1O</smiles>

Cyanidin 3-glucoside

Figure 1. Schematic representation of anthocyanin biosynthesis pathway. $P A L$, phenylalanine ammonia-lyase; $C 4 H$, cinnamate-4-hydroxylase; $C L$, 4-coumarate: CoA-ligase; $\mathrm{CHS}$, chalcone synthase; $\mathrm{CHI}$, chalcone isomerase; $\mathrm{F} 3 \mathrm{H}$, flavanone 3-hydroxylase; F3' $\mathrm{H}$, flavonoid 3'-hydroxylase; DFR, dihydroflavonol 4-reductase; LDOX, leucoanthocyanidin dioxygenase and UFGT, UDP-glucose: flavonoid 3-O-glucosyltransferase.

production of valuable and medicinally important plant products. However, the use of these cultures for commercial purposes is not favorable because of low productivity, cell line instability, fluid mixing, shear stress, and light transfer limitations. 9 A few studies have been reported to develop a protocol for the large-scale anthocyanin production, namely, Perilla frutescens cells grown in a $2.6 \mathrm{~L}$ stirred bioreactor, ${ }^{10} \mathrm{~V}$. vinifera callus grown in an airlift type bioreactor, ${ }^{11}$ and Vaccinium pahalae grown in a $10 \mathrm{~L}$ bioreactor. ${ }^{12}$ The effect of several factors such as culture medium parameters, elicitation, precursor feeding, and selection of cell lines on the anthocyanin accumulation in suspension and callus cultures has also been investigated. ${ }^{13}$

We had earlier demonstrated that the D. carota cell suspension cultures indicate stable growth under submerged conditions in shake-flask. Nitrogen $\left(\mathrm{NH}_{4} \mathrm{NO}_{3}\right.$ and $\left.\mathrm{KNO}_{3}\right)$ is a critical medium component that influences biomass accumulation and secondary metabolite production under in vitro culture conditions. ${ }^{14}$ Growing cultures in salt stress medium support the higher accumulation of secondary metabolites as reported previously with different ratios of $\mathrm{NH}_{4} \mathrm{NO}_{3}: \mathrm{KNO}_{3}$ for higher production of anthocyanin. ${ }^{15}$

The aim of the present work is to develop an in vitro $D$. carota cell suspension culture from flask levels (capacity of flask-100, 150, 250, 500, and $1000 \mathrm{~mL}$ ) to bioreactor levels $(3.5 \mathrm{~L})$ in the presence of salt stress in order to obtain stable and continuous anthocyanin production. To understand the influence of salt stress on the anthocyanin-generating cell line of $D$. carota on a different volumes, comparison studies were conducted at the flask and bioreactor levels, where cell growth, anthocyanin, phenolics, and flavonoid contents were assessed. To better understand the key gene engaged in augmentation of anthocyanin in D. carota cell culture, the expression levels of anthocyanin biosynthesis genes and putative anthocyanin transporter (MATE) involved in anthocyanin accumulation were quantified at the flask and bioreactor levels. The outcome of the study provides a better understanding of anthocyanin biosynthesis and transportation gene in flask and bioreactor under salt stress conditions.

\section{MATERIALS AND METHODS}

Initiation of Suspension Culture. The suspension culture of D. carota (Var. Atomic red) was established previously. ${ }^{15}$ Briefly, friable callus was induced from leaf explants of one-month-old seed-derived plantlets of D. carota on Murashige and Skoog (MS) medium containing 9.1 $\mu \mathrm{M} 2$, 4-dichlorophenoxyacetic acid (2, 4-D, Hi-media, India) and $2.32 \mu \mathrm{M}$ Kinetin (Kin, Hi-media, India). Pigmentation was initiated in callus inoculated in the anthocyanin induction medium containing indole acetic acid (11.4 $\mu \mathrm{M}$ IAA) and kinetin $(0.9 \mu \mathrm{M}$ Kin). Suspension cultures were established using $0.2 \mathrm{~g}$ pigment callus transferred to a $100 \mathrm{~mL}$ flask containing an anthocyanin induction medium. The $\mathrm{pH}$ of the medium was adjusted to 5.8 before autoclaving. The cultures were placed on a gyratory shaker $\left(24^{\circ} \mathrm{C} \pm 2,90 \mathrm{rpm}\right)$ under a 
$16 / 8 \mathrm{~h}$ photoperiod $\left(20-40 \mu \mathrm{mol} \mathrm{s}^{-1} \mathrm{~m}^{-2}\right)$ and were subcultured every 18 days.

Scale-Up of Anthocyanin Production. The cultures were maintained at $100,150,250,500$, and $1000 \mathrm{~mL}$ volume flask with $5 \mathrm{~g} \mathrm{~L}^{-1}$ of inoculum cell density to study growth and anthocyanin accumulation. The medium was supplemented with IAA and Kin and maintained in the light photoperiod of $16 / 8 \mathrm{~h}$. The flasks (liquid-phase culture) were incubated at 24 $\pm 2{ }^{\circ} \mathrm{C}$ in a gyratory shaker rotating at $90 \mathrm{rpm}$.

Stirred Tank Bioreactor. Previous studies have been conducted with callus culture of $D$. carota to optimize the salt stress level of $\mathrm{NH}_{4} \mathrm{NO}_{3}$ and $\mathrm{KNO}_{3}$ in the MS medium for higher anthocyanin production. ${ }^{15,16}$ The best medium composition achieved previously has been further taken in this study for scale-up anthocyanin production from the flask level $(150 \mathrm{~mL})$ to the bioreactor $(3.5 \mathrm{~L})$. The cultures were grown and maintained at the flask, level were used to scale-up to the bioreactor level under salt stress. The detailed composition of control and salt stress medium used for comparative studies of anthocyanin accumulation in the flask and bioreactor is shown in Table 1 .

Table 1. Composition of Control and Salt Stress Medium Used for Enhanced Production of Anthocyanin in Flask and Bioreactor Cell Suspension Culture of D. carota

\begin{tabular}{lcc}
\multicolumn{1}{c}{ composition } & control medium & salt stress medium \\
$\mathrm{NH}_{4} \mathrm{NO}_{3}$ & $20.0 \mathrm{mM}$ & $20.0 \mathrm{mM}$ \\
$\mathrm{KNO}_{3}$ & $18.8 \mathrm{mM}$ & $37.6 \mathrm{mM}$ \\
$\mathrm{IAA}$ & $11.41 \mu \mathrm{M}$ & $11.41 \mu \mathrm{M}$ \\
kin & $0.93 \mu \mathrm{M}$ & $0.93 \mu \mathrm{M}$ \\
inoculum density & $5 \mathrm{~g} \mathrm{~L}^{-1}$ & $5 \mathrm{~g} \mathrm{~L}^{-1}$ \\
medium pH & 5.8 & 5.8
\end{tabular}

The bioreactor was aerated through a stainless-steel sparger at an airflow rate of $0.1 \mathrm{vvm}$. The impeller's rotational speed (marine propeller-a low shear impeller) in the reactor was kept constant at $90 \mathrm{rpm}$ throughout the cultivation. The $\mathrm{pH}$ and temperature in the reactor were maintained at 5.8 and 24 $\pm 2{ }^{\circ} \mathrm{C}$, respectively. The cultivation was done under a $16 / 8 \mathrm{~h}$ photoperiod condition. The light for the reactor was provided using six halogen bulbs connected individually to the electrical supply, such that the light intensities could be varied in the range of $40-50 \mu \mathrm{mol} \mathrm{s} \mathrm{s}^{-1} \mathrm{~m}^{-2}$. During the culture period, samples were withdrawn from the reactor in 3 days till 18 days and analyzed for fresh weight, growth index, total anthocyanin content, total phenolic content, total flavonoid content, and isolation of RNA.

Determination of Fresh Weight and Growth Index. The culture was harvested at an interval of every 3 days after inoculation during 18 days of the culture period. The cells were washed with sterile water, separated by centrifugation, weighed (fresh weight) on a weighing balance, and blotted dry. The moisture content was analyzed using the fresh weight and dry weight of callus according to the method of Chevala et al. ${ }^{17}$ In the callus cells, the moisture content was measured and found to be $80-85 \%$. The growth index (GI) was calculated as follows

$$
\begin{aligned}
& \text { growth index (\% per day) } \\
& \qquad=\frac{\text { final weight }(\mathrm{g})-\text { initial weight }(\mathrm{g})}{\text { initial weight }(\mathrm{g})} \times 100
\end{aligned}
$$

Extraction Protocol and Quantification of Anthocyanin. The callus was separated from the medium by centrifugation (Hettich Universal 320R centrifuge, Germany) for total anthocyanin content estimation. The extraction of anthocyanin was performed as per the protocol described by Ji et al. $^{18}$ with minor modifications. Briefly, the callus was homogenized in the extraction buffer $[0.1 \%(\mathrm{v} / \mathrm{v})$ hydrochloric acid $(\mathrm{HCl})$-methanol] using a pestle and mortar; then, it was extracted by shaking overnight on a mechanical shaker at $4{ }^{\circ} \mathrm{C}$ in the dark. The homogenate was centrifuged for $5 \mathrm{~min}$ at $13,000 \times g$, and the upper aqueous phase was subjected to spectrophotometric quantification at $535 \mathrm{~nm}$ using an ultraviolet-visible (UV-vis) spectrophotometer (UV 1800 Shimadzu, Japan). The total anthocyanin content was expressed as $\mathrm{mg} \mathrm{L}^{-1}$ of fresh weight (FW).

Quantification of Total Phenolic and Flavonoid Content. The total phenolic content of samples was estimated using the calorimetric method rendering to the FolinCiocalteu (FC) assay. ${ }^{19}$ Briefly, a known volume of extract was mixed with FC reagent and after 5 min of incubation, sodium carbonate solution $(20 \% \mathrm{w} / \mathrm{v})$ was added to the solution. Finally, the mixed solution was allowed to stand for 1 $\mathrm{h}$ at room temperature, and the absorbance was measured at $765 \mathrm{~nm}$ in UV-Vis spectrophotometer. Total phenolic content was calculated as mg of gallic acid equivalent per $100 \mathrm{~g}$ of FW by using an equation acquired from the gallic acid calibration curve. Total flavonoid content was measured with the method reported by Anjum et al. ${ }^{20}$ Briefly, the extract was mixed with a known volume of potassium acetate $(1 \mathrm{M})$ and $\mathrm{AlCl}_{3}(10 \%, \mathrm{w} /$ v). This mixture was incubated for $30 \mathrm{~min}$ at room temperature after the addition of distilled water. The absorbance of the reaction mixture was measured at $415 \mathrm{~nm}$ by using UV-Vis spectrophotometer. Rutin was used as a standard for plotting the calibration curve, and the total flavonoid content was expressed as rutin equivalents per $100 \mathrm{~g}$ of FW.

Determination of Transcript Levels by qRT-PCR. The qRT-PCR analysis was conducted to study the influence of salt stress on the anthocyanin biosynthesis genes at flask and bioreactor. All the primers used for the differential expressions are listed in Supporting Information Table S1. The RNA was isolated from callus cultures using the cetyltrimethylammonium bromide (CTAB) method ${ }^{21}$ and was subjected to DNase I (Thermo Fischer Scientific India Pvt. Ltd, Bengaluru) treatment for the removal of deoxyribonucleic acid (DNA). Subsequently, the c-DNA synthesis kit (Thermo Fischer Scientific India Pvt. Ltd, Bengaluru) was used for reverse transcription. c-DNA isolated from callus cultures grown in control medium served as control. The transcript level of the culture grown in the flask and bioreactor with a salt stress medium was quantified using the quantitative real timepolymerase chain reaction ( $\mathrm{qRT}-\mathrm{PCR}$ ). The reaction mixture $(20 \mu \mathrm{L})$ contained cDNA, primers, and SYBR Premix Ex Tag (Takara, Bengaluru). The qRT-PCR analysis was performed by the Quant studio 5 (Applied Biosystem, India) using the following program: denaturation at $95^{\circ} \mathrm{C}$ for $30 \mathrm{~s}$, at $95^{\circ} \mathrm{C}$ for $5 \mathrm{~s}$, and at $58{ }^{\circ} \mathrm{C}$ for $30 \mathrm{~s}$. The relative expression levels were calculated using the comparative CT method. All the real-time PCR quantifications were performed with a nontemplate control and the endogenous control actin $(A C T)$, tubulin (TUB), and glyceraldehyde-3-phosphate dehydrogenase $(G A P D H)$. The relative expression data were calculated by 


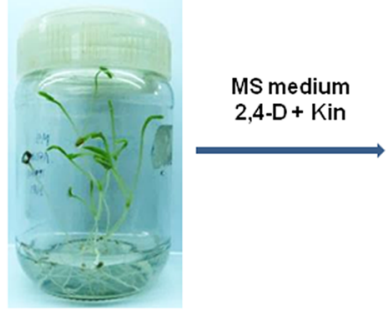

(a)

D. carota seedlings

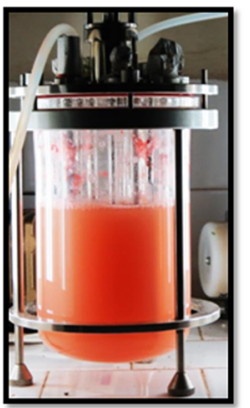

(g)

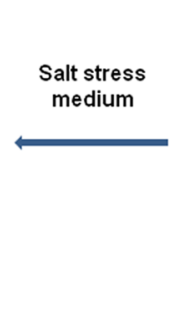

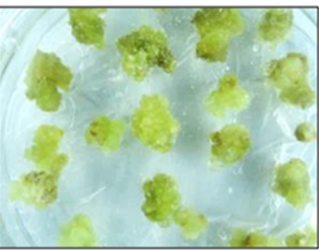

(b)

Induction of callus

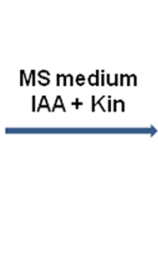

$\square$

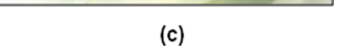

(c)

Selection of high yield cell line

MS medium IAA + Kin

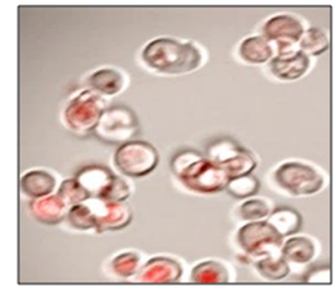

(f)

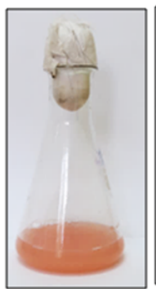

(e)

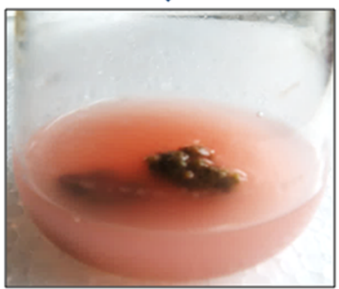

(d)

Stirred tank bioreactor

Cell suspension stage

Figure 2. Establishment of D. carota callus and cell suspension culture. (a) In vitro propagated plantlet served as explant, (b) callus has grown in the medium with 2,4-D and Kin, (c) anthocyanin accumulation in callus culture, (d) initiation of suspension culture, (e) suspension culture grown in the shake flask, (f) confocal microscopy picture of anthocyanin containing cells, and ( $\mathrm{g}$ ) culture grown in the bioreactor with salt stress medium.

the $2^{-\Delta \Delta C t}$ method, ${ }^{22}$ and the experiment was performed in two technical replicates and three biological samples.

Selection of Candidate Reference Genes of qRT-PCR. Three candidate reference genes (ACT, TUB, and GAPDH) were selected for evaluation to determine their suitability for the use to normalize the expression level of anthocyanin biosynthesis genes from $D$. carota cell culture grown in flask and bioreactor through qRT-PCR.

Statistical Analysis. The results were examined by the analysis of variance (ANOVA) using the SPSS 23 software. The data are the mean of three individual analyses and presented as a standard deviation. The post-hoc Tukey's test was used to analyze statistical differences between the samples. A value of $P<0.05$ was considered significant. The correlation between biomass, growth index, phenolic, flavonoids, and anthocyanin content was estimated using Pearson's correlation coefficient at a $P$-value $<0.05$ was considered significant. To normalize the candidate reference genes, NormFinder and geNorm algorithms ${ }^{23}$ were used to evaluate the expression stability and selection of reference genes. Finally, the qRT-PCR data normalization was selected based on ranked stability values (M) obtained from NormFinder and geNorm. The heatmap of the qRT-PCR expression data was constructed using the "complex heatmap" package in $\mathrm{R}$ studio. The comparative $\Delta \Delta C T$ method was used to calculate the relative expression levels of the different genes, and log fold change was expressed in the heatmap and enhanced volcano plot.

\section{RESULTS}

Establishment of Suspension Culture. The production of anthocyanin was initiated by the inoculation of $D$. carota seeds into a basal MS medium. The seedlings were grown during 2-3 weeks of inoculation at $24 \pm 2{ }^{\circ} \mathrm{C}$ (Figure 2a) in the dark. These seedlings were used to induce callus into a medium containing 9.1 $\mu \mathrm{M} 2,4-\mathrm{D}$, and $2.32 \mu \mathrm{M}$ Kin (Figure b). Callus emerged within 3-4 weeks of inoculation with the granular appearance and greenish in color, which was subcultured in the fresh medium after 4 weeks of interval.

The accumulation of anthocyanin in the medium comprising $11.41 \mu \mathrm{M}$ IAA and $0.93 \mu \mathrm{M}$ Kin was initiated by a 1 -monthold green and friable callus (Figure 2c). Green callus was maintained and sub-cultured in the anthocyanin induction medium (IAA and Kin) for 6-7 sub-cycles for initiation of pigmentation. Fully grown pigmented callus were further used for the establishment of suspension culture (Figure 2d,e). The culture was maintained in a liquid anthocyanin induction medium for continuous production of pigmentation and subcultured after 18 days of inoculation. Confocal fluorescence microscopy was utilized to show the accumulation of pigment inside the cytoplasm in induced suspension cells (Figure $2 \mathrm{f}$ ). The autofluorescence in the red spectrum $(\chi=458,488$, and $514 \mathrm{~nm}$ ) was strongly associated with the localization of anthocyanin in the cells. Further, pigmented callus grown in anthocyanin induction medium were transferred to suspension medium and maintained for one year in shake flask. The actively growing cell culture of $D$. carota was transferred to a 3.5 L stirred tank bioreactor with salt stress medium mentioned in Table 1 . The maximum production of anthocyanin was achieved on day 9 in the bioreactor (Figure $2 \mathrm{~g}$ ).

Scale-Up of Anthocyanin Production through Flask to Bioreactor Level. For the commercialization of secondary metabolites production, the scale-up from the flask level to a bioreactor is the critical step. To study the growth pattern and anthocyanin accumulation in the flask culture, the cell suspension was gradually sub-cultured from 100, 150, 250, 500 to $1000 \mathrm{~mL}$ flask into MS media containing $11.41 \mu \mathrm{M}$ IAA and $0.93 \mu \mathrm{M}$ Kin. Figure 3 describes the biomass and 

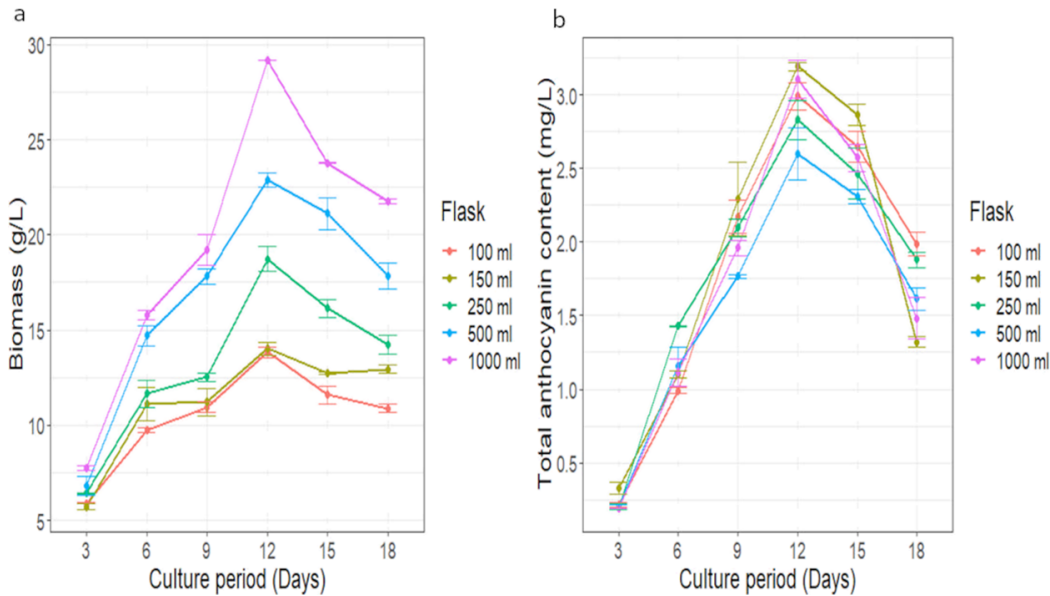

Figure 3. Time course of (a) biomass and (b) anthocyanin accumulation in D. carota cell cultures grown in different-sized vessels in the medium containing IAA and Kin. Each value represents the mean \pm SD in three replicates of an individual experiment.

anthocyanin production profile in different volume flasks. The suspension cells inoculated in the medium underwent a lag phase for 3 days followed by an exponential phase where they started increasing in numbers; a cell elongation phase took place, leading to a substantial increase in the fresh weight on day 3; this continued until day 12 when the maximum biomass and anthocyanin accumulation occurred, followed by a stationary phase characterized by a cessation of cell growth. The growth kinetics study in the flasks showed a reduction in cell growth from 15 days of inoculation. It was observed that in the flasks, the cell growth was synchronous and displayed the highest biomass of $13.83 \pm 0.38,14.03 \pm 0.48,18.74 \pm 0.91$, $22.86 \pm 0.52$, and $29.16 \pm 0.01 \mathrm{~g} \mathrm{~L}^{-1}$ in the $100,150,250,500$, and $1000 \mathrm{~mL}$ respectively on day 12 , and thereafter, a steep decline in cell growth was observed (Figure 3a).

Figure $3 \mathrm{~b}$ shows the total anthocyanin content in the different-volume flask. An increase in the anthocyanin accumulation in suspension cells was observed till day 12, followed by a decline in production from day 15 onwards. The anthocyanin contents in different-volume flask were $\sim 2.99 \pm$ $0.13,3.19 \pm 0.04,2.83 \pm 0.19,2.60 \pm 0.25$, and $3.11 \pm 0.18$ $\mathrm{mg} \mathrm{L}^{-1} \mathrm{FW}$ in the $100,150,250,500$, and $1000 \mathrm{~mL}$ flask, respectively.

Comparison of Cell Growth and Metabolite Production in Flask and Bioreactor Level under Salt Stress. The growth kinetics were examined based on biomass and growth index along with metabolite production in $D$. carota cell culture during the culture period of 18 days in the flask $(150 \mathrm{~mL})$ and bioreactor $(3.5 \mathrm{~L})$ culture with control and salt stress medium. Biomass, growth index, and total anthocyanin content in the cell culture were recorded for a period of 18 days with a 3-day interval (Figure 4). In flask culture, the lag phase was shorter at the 3rd day, and later, it reached the log phase, which started from day 3 and reached up to 12 days in a culture grown with control medium as well as in salt stress medium. The growth of cells started to decline from day 15 in both control and salt stress mediums. The suspenion cell cultures were initiated with $5 \mathrm{~g} \mathrm{~L}^{-1}$ of inoculum cell density which reached maximum biomass was displayed by the control $\left(31.97 \pm 0.37 \mathrm{~g} \mathrm{~L}^{-1} \mathrm{FW}\right)$ and salt stress medium $\left(27.97 \pm 1.04 \mathrm{~g} \mathrm{~L}^{-1} \mathrm{FW}\right)$ during $\log$ phase (day 12) of the culture period (Figure 4a).

The growth curve of the cells grown in the bioreactor indicated that the growth was initially slow during the first 3 days in the control and salt stress medium. However, as the cultures proceeded, it showed a marked increase from day 6 onward and accumulated a great amount of biomass at day 12 of the culture period. The maximum increase in biomass was reached on day 12 , which was $45.93 \pm 0.28$ and $38.15 \pm 0.38 \mathrm{~g}$ $\mathrm{L}^{-1} \mathrm{FW}$ in the control and salt stress medium, respectively (Figure 4b). In comparison to flask culture, the growth of cells was higher in a bioreactor which was 1.43 -fold in the control medium and 1.36-fold in the salt stress medium.

However, the growth index of the flask and bioreactor culture showed a similar response with control and salt stress condition where growth kinetics with exponential phase lasted from day 3 to 12 followed by a stationary phase (until 18 days). The growth of cell culture was maintained from $60.67 \pm$ 1.74 to $84.36 \pm 0.18 \% \mathrm{~d}^{-1}$ in the control medium and $57.57 \pm$ 1.65 to $82.11 \pm 0.67 \% \mathrm{~d}^{-1}$ in the salt stress medium throughout the culture period of flask culture (Figure 4c). However, the maximum growth index was $89.11 \pm 0.07 \% \mathrm{~d}^{-1}$ on day 12 in the control medium, whereas it was $86.89 \pm$ $0.13 \% \mathrm{~d}^{-1}$ on day 12 in the salt stress medium in the bioreactor (Figure $4 \mathrm{~d}$ ). The growth index of the control and salt stress medium in the bioreactor was $79.97 \pm 0.09 \% \mathrm{~d}^{-1}$ in the control and $68.10 \pm 1.11 \% \mathrm{~d}^{-1}$ at the end of the growth period (18 days).

The present results have shown that the total anthocyanin content of D. carota cell culture in the flask level was also affected by salt stress during the culture period (Figure $4 \mathrm{e}$ ). The maximum anthocyanin content was recorded in a salt stress medium with $4.33 \pm 0.19 \mathrm{mg} \mathrm{L}^{-1} \mathrm{FW}$ on day 9, whereas $2.18 \pm 0.19 \mathrm{mg} \mathrm{L}^{-1} \mathrm{FW}$ was observed in the control medium on day 12 . On the other hand, total anthocyanin content in the bioreactor with the control and salt stress medium is shown in Figure $4 \mathrm{f}$. Compared to the control medium, higher anthocyanin production was seen in the salt stress medium. In the control medium, the anthocyanin content obtained was $4.55 \pm 0.30 \mathrm{mg} \mathrm{L}^{-1} \mathrm{FW}$, which was 2.09 -fold higher than flask culture grown in the control medium, while the salt stress medium showed a $10.91 \pm 1.03 \mathrm{mg} \mathrm{L}^{-1} \mathrm{FW}$, which is 2.52 -fold higher than the flask culture grown in salt stress medium.

Total Flavonoid and Phenolic Content in Flask and Bioreactor Level under Salt Stress. Like anthocyanin content, control and, salt stress medium have shown a similar flavonoid and phenolic content in the flask and bioreactor level (Figure 5). During flask culture, the phenolic content was higher at day $12(32.65 \pm 1.18 \mathrm{mg} / 100 \mathrm{~g} \mathrm{FW})$ in the control 
a
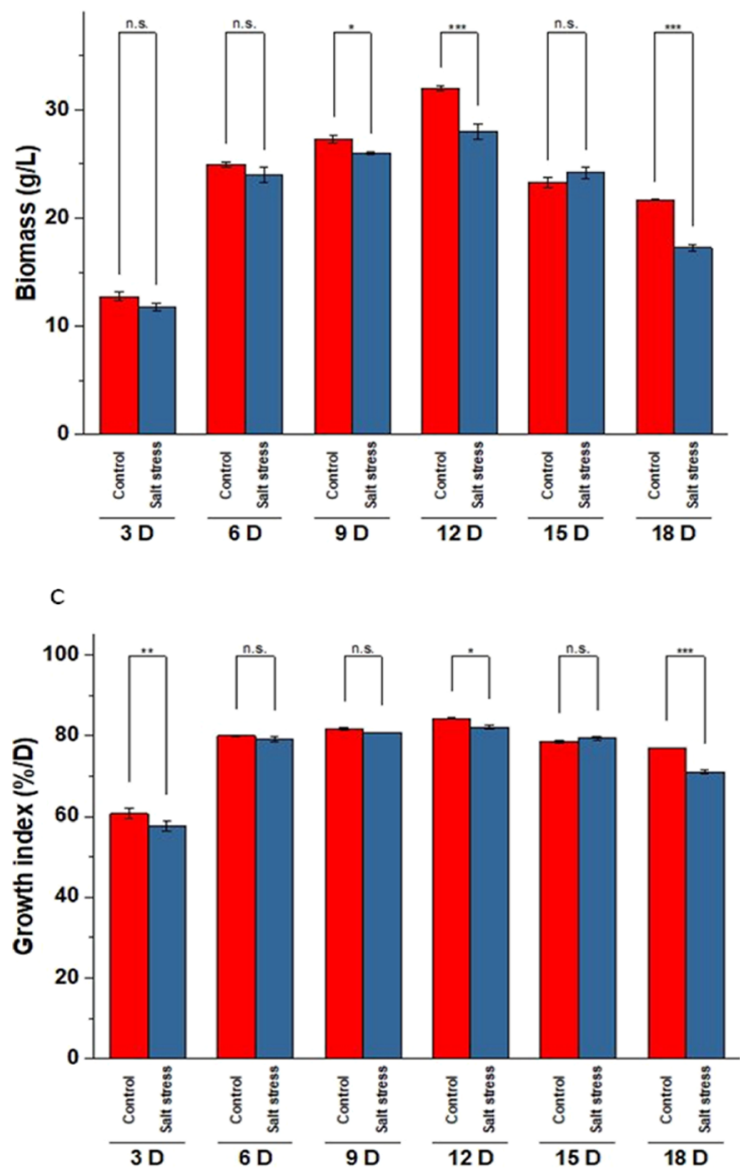

e

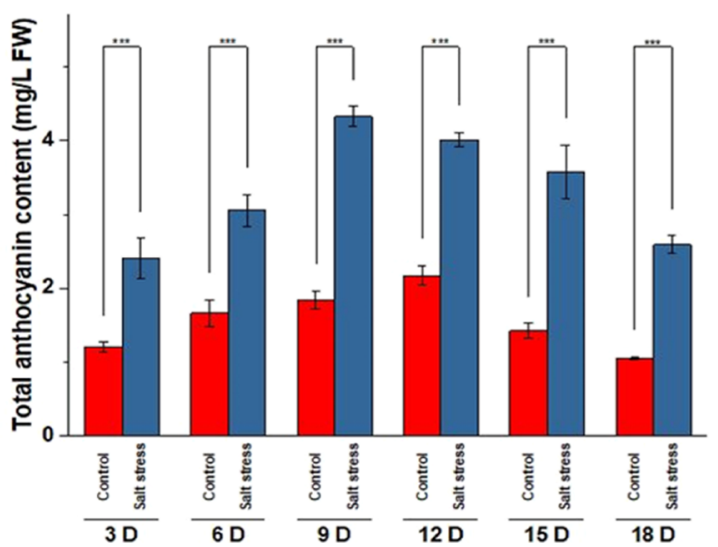

b

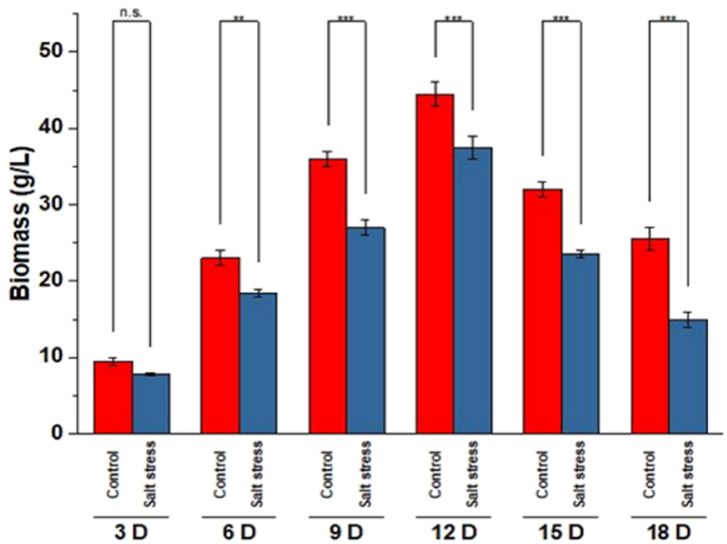

d

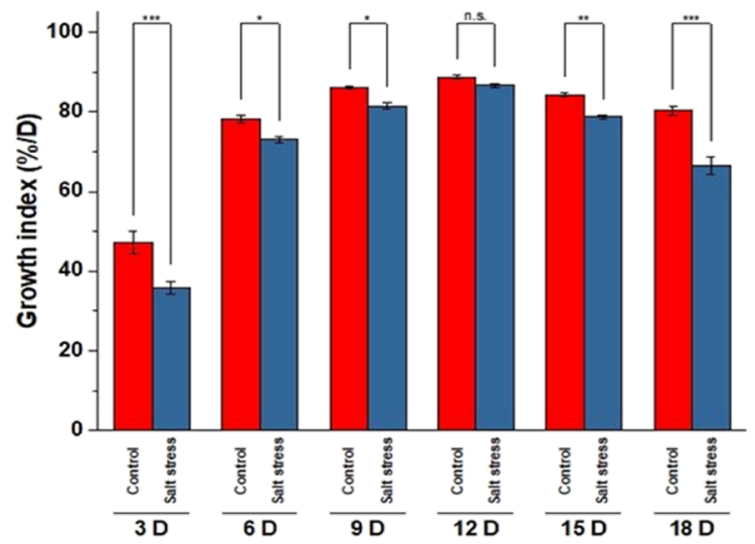

f

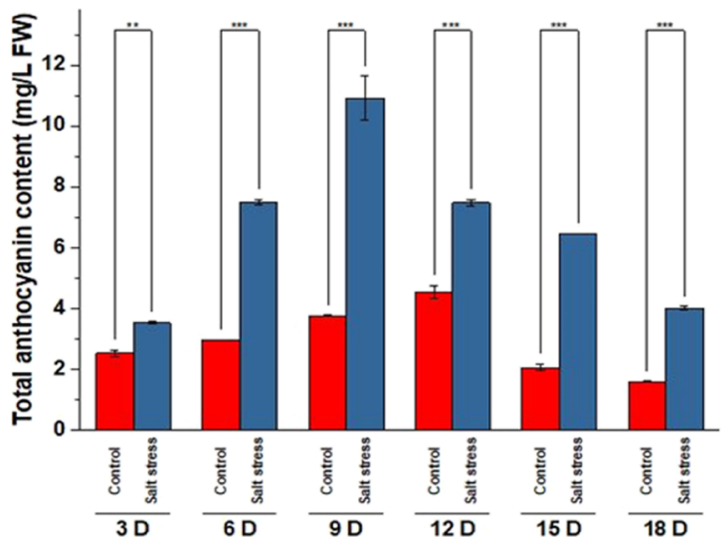

Figure 4. Comparative study of biomass, growth index, and total anthocyanin content in the flask (a,c,e) and bioreactor (b,d,f) cell culture of $D$. carota in response to salt stress. Data are represented as the mean \pm standard deviation from three replicates. The significance level among control and salt stress medium indicated as ns, $P>0.05, * P \leq 0.05, * * P \leq 0.01, * * * P \leq 0.001$.

medium and day $9(49.41 \pm 0.99 \mathrm{mg} / 100 \mathrm{~g} \mathrm{FW})$ in the salt stress medium, respectively (Figure 5a). On the other hand, bioreactor culture grown in salt stress indicated maximum accumulation of phenolic content on day $9(132.79 \pm 1.25$ $\mathrm{mg} / 100 \mathrm{~g} \mathrm{FW})$, whereas, in the control medium, the highest phenolic content of $59.15 \pm 1.10 \mathrm{mg} / 100 \mathrm{~g} \mathrm{FW}$ was observed on day 12 (Figure $5 \mathrm{~b}$ ). Furthermore, in comparison to flask culture, bioreactor culture showed 2.69-fold higher phenolic content than the flask with salt stress medium.
In flask culture, total flavonoid content was obtained as $28.96 \pm 0.49$ with control medium at day 12 and $43.01 \pm 1.12$ $\mathrm{mg} / 100 \mathrm{~g} \mathrm{FW}$ with salt stress medium at day 9 (Figure 5c). During bioreactor culture, the highest accumulation of flavonoid content $(84.58 \pm 1.70 \mathrm{mg} / 100 \mathrm{~g} \mathrm{FW})$ was observed in cells grown in the salt stress medium for 9 days, which was almost 1.89-fold higher than control medium $(43.21 \pm 3.39$ $\mathrm{mg} / 100 \mathrm{~g} \mathrm{FW})$. The total flavonoid content in cell culture of the bioreactor level reached the highest value on days 12 and 9, which was 1.54- and 1.97-fold higher than those in the flask 

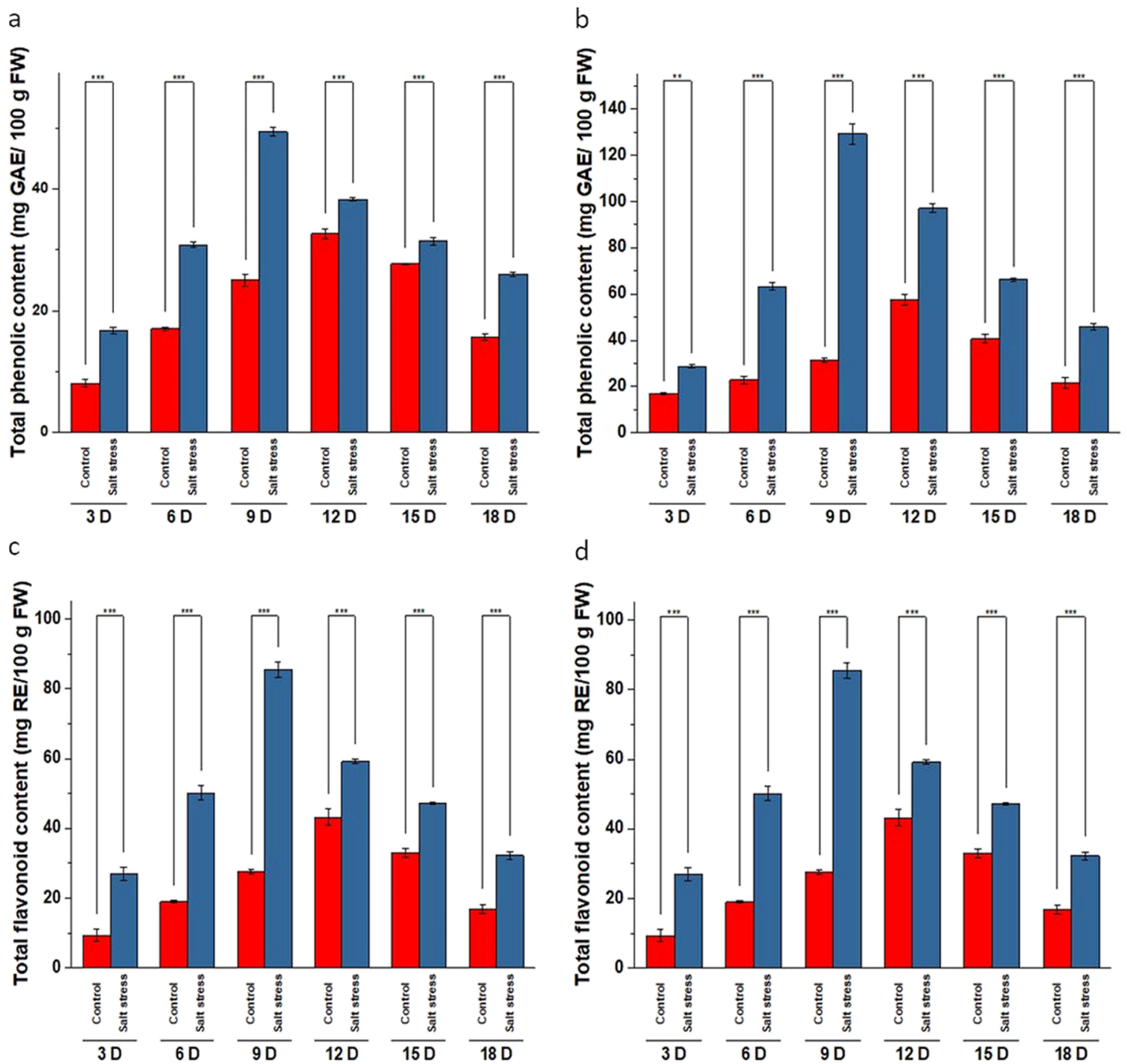

Figure 5. Comparative study of total phenolic and flavonoid content in the flask $(\mathrm{a}, \mathrm{c})$ and bioreactor $(\mathrm{b}, \mathrm{d})$ cell culture of $D$. carota in response to salt stress. Data are represented as the mean \pm standard deviation from three replicates. The significance level among control and salt stress medium indicated as $\mathrm{ns}, P>0.05, * P \leq 0.05, * * P \leq 0.01, * * * P \leq 0.001$.

with control and salt stress medium respectively (Figure 5d). Subsequently, a significant decline in flavonoid content was noted with the culture period because cell growth was switching from exponential phase to stationary phase.

The Pearson correlation coefficient was determined between cell growth and metabolite production in the presence of a salt stress medium at flask and bioreactor level. In flask culture, the results showed a positive correlation between the content of total phenols and total flavonoids $\left(R^{2}=0.971, P<0.01\right)$. However, a positive correlation was also found between total flavonoids and anthocyanin $\left(R^{2}=0.793, P<0.01\right)$, and phenolic and anthocyanin $\left(R^{2}=0.810, P<0.01\right)$, indicating that high concentrations of total flavonoids and phenolic were accompanied by the high concentration of anthocyanin in the cell culture of D. carota (Table 2).

During bioreactor culture, analysis showed that there were significant correlations between total anthocyanin content (TAC) and total flavonoid content (TFC) $\left(R^{2}=0.940, P<\right.$ $0.01)$ and between total anthocyanin content and total phenolic content $\left(R^{2}=0.937, P<0.01\right)$. Also, flavonoid and phenolic content also showed a significant correlation $\left(R^{2}=\right.$
Table 2. Pearson Correlation Coefficients Between Biomass, Growth Index, Total Phenolic, Flavonoid, and Anthocyanin Content in Suspension Culture Grown With Control and Salt Stress Medium in Flask $(n=24)^{a, b, c}$

\begin{tabular}{lccccc}
\multicolumn{5}{c}{ correlation coefficient $(r)$} \\
& FW & GI & TAC & TFC & TPC \\
FW & & $0.964^{* *}$ & 0.327 & $0.696^{* *}$ & $0.636^{* *}$ \\
GI & & 0.288 & $0.650^{* *}$ & $0.615^{* *}$ \\
TAC & & & $0.793^{* *}$ & $0.810^{* *}$ \\
TFC & & & & $0.971^{* *}$
\end{tabular}

TPC

${ }^{a} \mathrm{FW}$, fresh weight; GI, growth index; TAC, total anthocyanin content; TFC, total flavonoid content; TPC, total phenolic content. ${ }^{b}$ *Correlation is significant at 0.05 level (two-tailed). ${ }^{c * *}$ Correlation is significant at 0.01 level (two-tailed).

0.982, $P<0.01)$, indicating that with the accumulation of anthocyanin, there will be a positive enhancement of flavonoids and phenolic content in suspension culture of $D$. carota (Table 3). 
Table 3. Pearson Correlation Coefficients Between Biomass, Growth Index, Total Phenolic, Flavonoid, and Anthocyanin Content in Suspension Culture Grown in Control and Salt Stress Medium in Bioreactor $(n=24)^{a, b, c}$

\begin{tabular}{|c|c|c|c|c|c|}
\hline \multicolumn{6}{|c|}{ correlation coefficient $(r)$} \\
\hline & FW & GI & TAC & TFC & TPC \\
\hline FW & & $0.882 * *$ & 0.168 & 0.359 & 0.0 .354 \\
\hline GI & & & 0.252 & $0.406^{*}$ & 0.404 \\
\hline TAC & & & & $0.940 * *$ & $0.937 * *$ \\
\hline TFC & & & & & $0.982 * *$ \\
\hline
\end{tabular}

${ }^{a} \mathrm{FW}$, fresh weight; GI, growth index; TAC, total anthocyanin content; TFC, total flavonoid content; TPC, total phenolic content.

$b_{*}$ Correlation is significant at the 0.05 level (two-tailed). $c * *$ Correlation is significant at the 0.01 level (two-tailed).

Expression of Reference Genes of $D$. carota under Salt Stress. We choose $A C T, T U B$, and GAPDH reference genes to normalize the expression data. Figure 6 shows

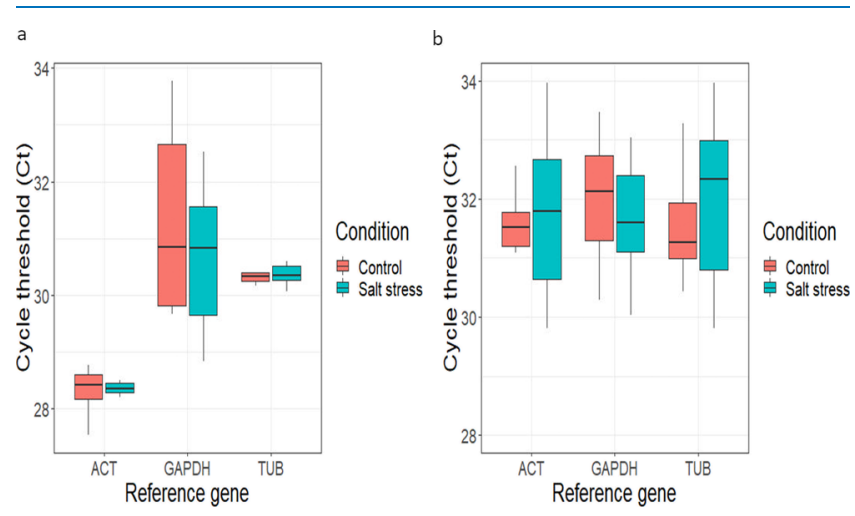

Figure 6. Box plots indicating the range of cycle threshold $(\mathrm{Ct})$ values of candidate reference genes from $D$. carota cell cultures grown in (a) flask and (b) bioreactor with control and salt stress medium. Candidate reference genes include $A C T, T U B$, and GAPDH. Values of three biological replicates taken as averages, whiskers represent the maximum and minimum values, and the median is depicted by the line across the box.

expression levels of the reference genes in the flask (Figure 6a) and bioreactor (Figure $6 \mathrm{~b}$ ) level with box plots that summarize the data $(n=18)$ for each reference gene. Each box represents the individual reference gene expression in the different time intervals of 3, 6, 9, 12, 15, and 18 days. A box plot comprises whiskers that represent the minimum and maximum values and the line across the box depicts the median. A marker shows the average cycle threshold $(\mathrm{Ct})$ value. A ranking (Supporting Information Tables S2 and S3) and stability value (Supporting Information Figures S1 and S2) of references genes in different samples of $D$. carota cell culture was also calculated to determine the optimal number of reference genes required for normalization of qRT-PCR data.

Comparison of Transcript Abundance of Anthocyanin Biosynthesis Genes in Flask and Bioreactor Level under Salt Stress. To analyze the expression of anthocyanin biosynthesis genes at the flask and bioreactor level, cells were harvested every 3 days of the interval from control and salt stress medium until 18 days of the culture period. The gene expression data were transformed to $\log 2$ fold change and presented with clusters using log 2 -fold change values as in heatmap of cell culture of flask and bioreactor level using color keys. The heatmap and hierarchical clustering analysis showed dynamic changes in $D$. carota cell cultures in shake flasks for anthocyanin biosynthesis (Figure $7 \mathrm{a}, \mathrm{c}$ ). The clustering analysis showed three main groups (day 6 and 9; day 15 and 18; day 3 and 12) according to the culture period and two additional clusters. However, the clustering tree is showing the separation of gene expression in the bioreactor based on culture period into two broad groups: 3 and 15 days comprise one group and 9 and 12 days comprise the second group. Further, in the first group, 3 and 15 days were clustered together with 18 days, and the second group, 9 and 12 days were clustered with 6 days of the culture period (Figure $7 \mathrm{~b}, \mathrm{~d}$ ). This shows that the expression of most of the genes was higher at day 9 in bioreactor culture. Like the bioreactor cluster, the flask culture also sheds a higher abundance of genes on day 9 of the culture period under salt stress.

The transcript level of anthocyanin biosynthesis genes has been shown to be induced by salt stress at both flask and bioreactor levels (Figure 7). On day 9, the expression level of PAL increased 2.91-fold in the flask culture and 2.67-fold in the bioreactor culture under salt stress as compared to the control medium, showing almost the same expression. The transcript level of $\mathrm{C} 4 \mathrm{H}$ was obtained as 2.54- and 5.90-fold higher in the flask and bioreactor with salt stress medium, respectively, compared to control. Notably, $\mathrm{CL}$ and $\mathrm{CHS}$ transcripts were observed as 5.04- and 4.06-fold in the flask culture, whereas the transcript level in the bioreactor culture was as high as 5.27- and 9.45-fold under salt stress, respectively. The transcript level of DFR showed an almost similar expression in the flask compared to the bioreactor as 3.68 and 3.25-fold, respectively. In contrast, the transcript abundance of $\mathrm{F} 3 \mathrm{H}$ was slightly enhanced by 2.76 -fold in the flask and 3.73-fold in the bioreactor level in response to salt stress. On the other hand, LDOX showed transcript levels of 3.91- and 4.41-fold in the flask and bioreactor, respectively, with the salt stress medium. However, UFGT peaked in the flask culture on day 9 as 8.31 -fold. The UFGT showed the highest transcript abundance in the bioreactor with the salt stress medium on day 9, which was 18.51-fold higher in the bioreactor with the salt stress medium. As shown in Figure 7, the cells grown in the salt stress medium showed a gradual increase in the transcript levels of all genes until day 9, after which they decreased.

Expression of Putative MATE Genes in Flask and Bioreactor under Salt Stress. To elucidate the role of transporter genes (MATE) in the transportation of anthocyanin from the cytoplasm to vacuole, we evaluated the putative MATE gene in flask and bioreactor levels in the culture of $D$. carota under salt stress by RT-qPCR analysis. During the initial days of the culture period (day 3), the expression of MATE was hardly detectable in the flask (Figure 7a,c). Meanwhile, the expression of MATE had a stronger upregulation on day 9. RTqPCR analysis suggested that co-expression of the putative MATE gene was observed with anthocyanin biosynthesis genes ( $C L, C H S$, and UFGT) on day 9. The maximum expression of putative MATE genes were 2.32-fold in flask culture under salt stress.

The expression of the putative MATE gene was also analyzed to validate its role under salt stress at the bioreactor level. In this analysis, we found that the MATE gene was upregulated in response to salt stress. The expression of the putative MATE gene was repressed at an early stage (day 3 ) 

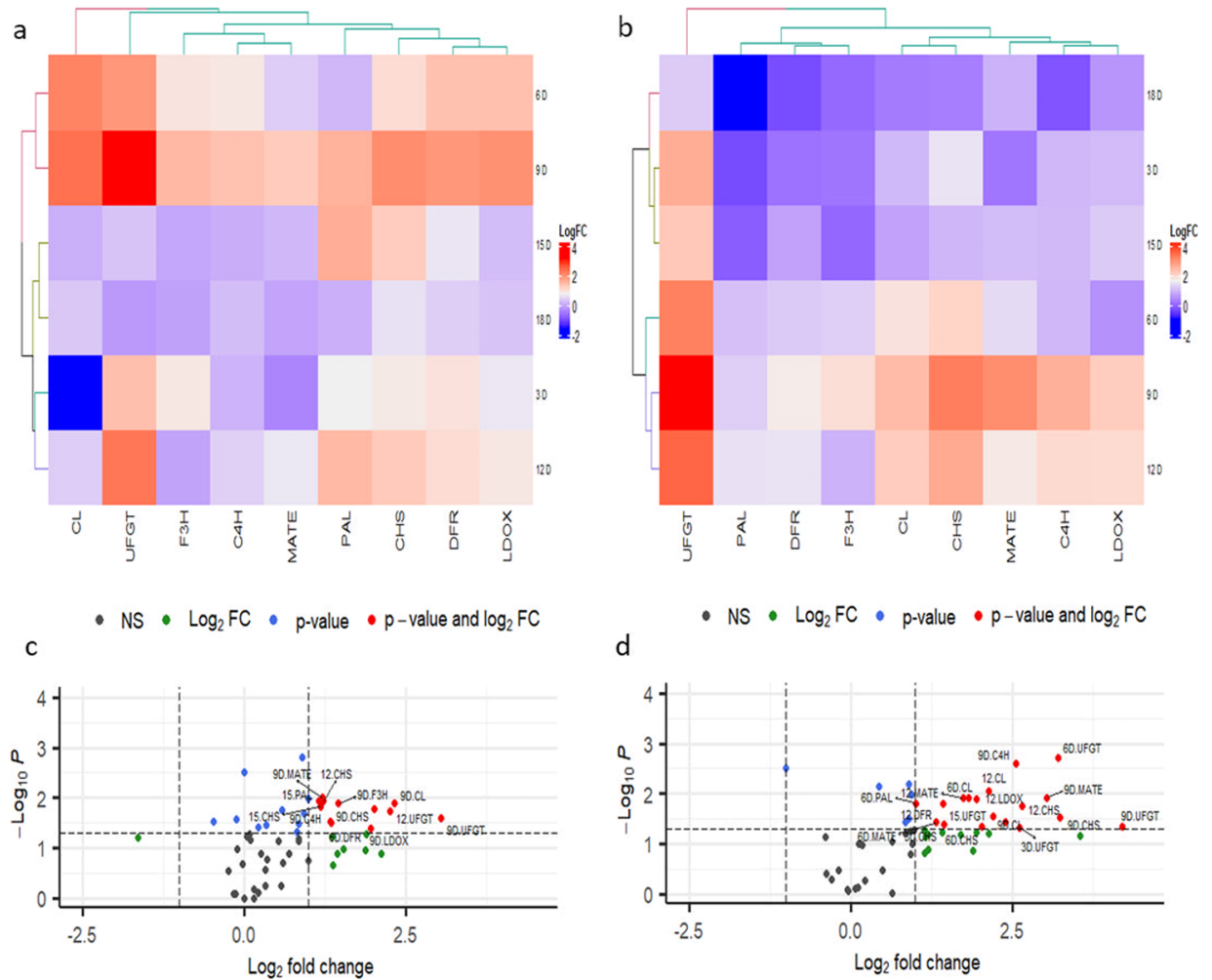

Figure 7. Gene expression comparison between cultures of $D$. carota cells grown in control and salt stress medium in flask and bioreactor levels. The heatmap shows the real-time quantitative RT-PCR analysis results with two technical and three biological replicates. The expression levels of genes are presented using fold-change values transformed to log 2 formats compared to control in the flask (a) and bioreactor (b). The color scale is relative to the abundance of each compound. Red indicates upregulation, and blue indicates downregulation. The volcano plot depicts differentially expressed genes in a flask (c) and bioreactor (d) based on $p$-value and log 2 (fold-change) at an $\alpha$ level of 0.05 and log FC cut off of 1.3. Colored dots correspond to individual genes whose expression differences were significant based on both $p$ and log FC value (red dots), only $p$ value (blue dots), only log FC (green dots), or not significant (gray dots) in either.

and later in the culture period (day 18) in a bioreactor. We found that the expression of MATE was significantly increased on day 9 of the culture period to 8.23 -fold, which was correlated with the anthocyanin biosynthesis gene $(\mathrm{C} 4 \mathrm{H}, \mathrm{CL}$, $\mathrm{CHS}$, and UFGT) under salt stress (Figure $7 \mathrm{~b}, \mathrm{~d}$ ).

\section{DISCUSSION}

The suspension culture of $D$. carota was initiated using pigmented callus into MS medium containing IAA and Kin. The homogenized cell suspension culture was obtained by regular sub-culturing in the same medium. The successful establishment of pigmented homogenized suspension culture was confirmed visually by obtaining pigmentation in suspension culture followed by confocal fluorescence microscopy. In vitro grown cell cultures changed enormously relying on the medium constituents, type of explant, plant growth hormone combinations, and culture environment conditions. MS medium constituents were seen as the best for the growth and development of the in vitro cell culture and furthermore for callus culture and secondary metabolite induction. Similar to the current studies, Rosa hybrida also showed anthocyanin induction in the presence of IAA and $\mathrm{Kin}^{24}$ The result obtained by Maharik et al. ${ }^{25}$ also showed that Kin along with NAA enhances the production of anthocyanin in the callus culture of Crataegus sinaica and 2, 4-D with Kin enhances anthocyanin in a Vitis sp. culture. ${ }^{26}$

In the present study, different volume flasks were employed for successful increase in anthocyanin accumulation, where maximal production attained at day 12 with mediumcontaining IAA and Kin in all the cultured flasks $[\sim 2.99 \pm$ $0.13(100 \mathrm{~mL}), 3.19 \pm 0.04(150 \mathrm{~mL}), 2.83 \pm 0.19(250 \mathrm{~mL})$, $2.60 \pm 0.25(500 \mathrm{~mL})$, and $3.11 \pm 0.18(1000 \mathrm{~mL}) \mathrm{mg} \mathrm{L}^{-1}$ FW)]. Our findings establish a gradual scaling up of anthocyanin production at the flask level (capacity 100 to $1000 \mathrm{~mL}$ flask) without depending on abiotic and biotic elicitors. Ceoldo et al. ${ }^{27}$ also pursued similar scale-up study of anthocyanin production in $D$. carota cell culture from a $250 \mathrm{~mL}$ flask and found that maximum anthocyanin was obtained on day $9(120 \mu \mathrm{g} / \mathrm{g} \mathrm{FW})$, whereas our findings achieved total anthocyanin contents of 404 and $166 \mu \mathrm{g} / \mathrm{g}$ FW in bioreactor as well as flask culture, respectively, under salt stress conditions.

Suspension culture was developed in D. carota cell suspension for anthocyanin synthesis in a $150 \mathrm{~mL}$ flask $^{28}$ and $250 \mathrm{~mL}$ flask in prior work. ${ }^{29}$ Narayan et al. ${ }^{30}$ produced a D. carota suspension culture in which anthocyanin levels declined steadily up to six subcultures, after which a $0.5 \mathrm{mg} \mathrm{L}^{-1}$ rise in IAA restored anthocyanin levels for a limited number of consecutive subcultures. However, scaling up anthocyanin synthesis from a flask to a bioreactor has not proven effective in in vitro cell cultures. Because of callus selection, stability, and de-differentiation, cell cultures are often diverse in cell composition, which can cause variation in growth rates when they are grown in large scale. However, the culture of Aralia cordata cell lines, which succeed and produce anthocyanin without light irradiation, was successfully scaled up from flasks to $500-\mathrm{L}$ pilot-scale bioreactors. ${ }^{31}$ The stable and continuous 
production of secondary metabolites from the flask level to bioreactor is typically not straightforward because of changes in the cell development condition regarding hydrodynamic shear powers and rheological properties. Fortunately, these limitations can be overcome by improving the bioreactor's design and optimizing the culture and system's key parameters such as adequate mixing, low-shear environment, nutrient availability, and reducing mass transfer for improved oxygen. ${ }^{32}$

The increase in anthocyanin content through the salt stress medium (20.0 $\mathrm{mM} \mathrm{NH}_{4} \mathrm{NO}_{3} / 37.6 \mathrm{mM} \mathrm{KNO}_{3}$ ), which was observed in the flask culture, was also confirmed in the stirred tank bioreactor culture. Salt stress medium constitutes were shown to be deleterious toward the growth of cells compared to the control medium. Remarkably, the growth index of the flask and bioreactor culture was almost similar. Consistent with the present study, a similar observation was also achieved by Jin et al. $^{33}$ during the finding of nitrogen concentration; it affects the biomass of Astragalus membranaceus adventitious roots. The current research is also supported by gymnemic acid and with withanolide A production in the presence of an increased level of $\mathrm{KNO}_{3}$ in the medium. ${ }^{34}$ The nitrogen source plays a major role in MS medium, which affects anthocyanin production by plant cell culture. This study revealed that the full strength of $\mathrm{NH}_{4} \mathrm{NO}_{3}(18.8 \mathrm{mM})$, and with double strength of $\mathrm{KNO}_{3}(37.6 \mathrm{mM})$ in the medium resulted in the improvement of anthocyanin accumulation in suspension culture, probably due to nutritional stress. Similarly, Echinacea angustifolia culture showed a higher ratio of $\mathrm{NH}_{4}^{+} / \mathrm{NO}_{3}{ }^{-}$ $(5: 25)$ is optimal for biomass and flavonoid accumulation. ${ }^{35}$

Based on the literature, various types of bioreactors have been utilized for large-scale production of plant cells including stirred-tank, air-lift, bubble column, membrane, wave, rotating drum, and hollow fiber bioreactors. Among them, stirred-tank bioreactors are the most commonly used bioreactors, offering a fairly simple scale-up, good fluid mixing, and oxygen transfer capability, and relatively easy conventionality with existing good manufacturing practices. ${ }^{32}$ Hence, the present study has chosen the stirred tank bioreactor for higher production of anthocyanin in suspension culture of $D$. carota under salt stress. Higher production of artemisinin was also reported with Artemisia annua in the modified stirred tank bioreactor using the optimized medium as compared with the shake flask cultivation. ${ }^{36}$ Shinde et al. ${ }^{37}$ have reported the importance of sucrose, phosphate, and nitrogen in the growth and production of the phytoestrogens daidzein and genistein by the suspension cultures of Psoralea corylifolia; here, a reduced concentration of phosphate $(0.625 \mathrm{mM})$ and a high ratio of the $\mathrm{NH}_{4}^{+}(20$ $\mathrm{mM}) / \mathrm{NO}_{3}{ }^{-}(40 \mathrm{mM})$ promoted the production of daidzein and genistein. On the other hand, a simultaneous increase in biomass and isoflavone accumulation was found upon a scaleup of the Cyclopia subternata suspension culture from shake flasks to stirred tank bioreactors.

To elucidate the role of salt stress on phenolics and flavonoid content in flask and bioreactor levels were examined. The maximum production of phenolics and flavonoid along with anthocyanin accumulation was obtained on day 9 in both flask and bioreactor levels. Moreover, phenolic and flavonoid production belongs to the polyphenol group of secondary metabolites, which are directly affected by anthocyanin accumulation. Similar to the present findings, Irshad et al. ${ }^{39 w}$ also found higher anthocyanin content exhibited higher total phenolic content and total flavonoid content, as well as enhanced antioxidant activity in callus culture of Abelmoschus esculentus. Thus, the findings of the present study suggest that anthocyanin contents positively affect the polyphenol content of the cell suspension cultures. The finding of our results is also supported by the result of Lulu et al. ${ }^{40}$ where phenolic and flavonoids yield was the highest at $\mathrm{NH}_{4}{ }^{+} / \mathrm{NO}_{3}{ }^{-}$ratio of $15: 30$ in the adventitious roots of Eurycoma longifolia through bioreactor culture system. Similarly, the findings of Cui et al. ${ }^{41}$ also found higher content of phenolic and flavonoid in the presence of higher levels of $\mathrm{NO}_{3}{ }^{-}$in the root culture of Hypericum perforatum. The abovementioned study confirms the easy, fast-growing, and higher-yielding anthocyanin production from a $100 \mathrm{~mL}$ flask culture to a $3.5 \mathrm{~L}$ stirred tank bioreactor under salt stress.

Quantitative RT-PCR is the most frequently used method for the quantification of transcript abundance in many plant species because it is a fast, sensitive, and accurate method. However, precise evaluation of relative gene expression via qRT-PCR depends upon the selection and use of suitable reference genes for expression normalization of target genes. ${ }^{42}$ Many reports have proved that the use of two reference genes compared to a single gene to get the most accurate and reliable normalization is adequate for studying gene expression. ${ }^{43}$ Hence, we have used three reference genes (ACT, TUB, and $G A P D H)$ for the normalization of biosynthesis gene expression.

In the present study, an attempt was made to elucidate the transcript level of anthocyanin biosynthesis genes $(P A L, C 4 H$, CL, CHS, DFR, F3H, LDOX, and UFGT) and putative transporter gene (MATE) in response to salt stress, the suspension culture of $D$. carota grown in the flask and bioreactor level was examined by qRT-PCR. Notably, the expression of CL, CHS, DFR, LDOX, and UFGT was continuously induced and maintained at a high level in the flask culture throughout the exponential phase ( 3 to 9 days), later declined with the death phase ( 12 to 18 days). While the expression of $C 4 H, C L, C H S, L D O X$, and UFGT was significantly showing a higher expression under salt stress in the bioreactor. The findings of Yin et al. ${ }^{26}$ also confirm the higher transcript level of UFGT with the modified medium constituent's concentration (such as starvation of phosphate) in the grape suspension cultures. The present study supports higher $\mathrm{KNO}_{3}$ levels in the medium, positively enhancing the anthocyanin content and their expression in both flask and bioreactor level. An increase in the supply of $\mathrm{KNO}_{3}$ in the medium activates the phenylpropanoid metabolism and production of phenolic compounds. ${ }^{4}$ Similar responses were reported in the expression of DFR, LDOX, and UFGT genes that were promoted under optimum conditions for anthocyanin accumulation in the suspension culture of Rosa hybrida. ${ }^{44}$

The expression profiles for the putative MATE gene throughout the culture period were correlated to the anthocyanin biosynthesis. During the culture period, from day 12 to 18 days, transcripts of the MATE gene were weakly present, while its expression was higher on day 9. Our work demonstrates the biochemical function of MATE proteins explicitly involved in the vacuolar transport of acylated anthocyanins. Plants have a many MATE proteins per species, suggesting that MATE proteins might play an essential role in the detoxification of secondary metabolites and xenobiotics in plants. ${ }^{45}$ To date, few MATE family members, which are localized at the tonoplast or the plasma membrane, have been functionally characterized. Gomez et al. ${ }^{46}$ have been identified the involvement of two grapevine AM1 and AM3 proteins 
mediate specifically acylated anthocyanin transport. Two MATE-type transporters VvMATE1 and VvMATE2 from $V$. vinifera $L$., have been identified as putative proanthocyanidin transporters during the development of berry. ${ }^{6}$ The present finding is also supported by the report by Gani et $\mathrm{al}^{47}$ that both vesicle trafficking and MATE transporter-mediated mechanisms are involved in the sequestration of anthocyanins to vacuoles. Our findings indicate that anthocyanin-type MATE transporter genes might be involved in the movement of anthocyanin across cell membranes, providing essential information for understanding the process underlying anthocyanin accumulation in D. carota cell culture.

\section{CONCLUSIONS}

In conclusion, $D$. carota culture was established up via the flask level and maintained up to a $3.5 \mathrm{~L}$ bioreactor under salt stress media to increase anthocyanin accumulation and acquire insight into its biosynthesis and transportation genes. In vitro anthocyanin synthesis in bioreactors has been reported in many articles, but the feasibility to produce in large scale has not been successful achieved. Furthermore, comparison experiments between the flask and bioreactor were carried out to examine the impact of medium salt components on cell development, phenolics, flavonoids, and anthocyanin content, with salt stress medium showing a significant improvement over the control conditions in the flask and bioreactor. The expression analysis revealed the co-expression of anthocyanin biosynthesis ( $\mathrm{C} 4 \mathrm{H}, \mathrm{CL}, \mathrm{CHS}$, and UFGT) and putative transporter gene (MATE) under salt stress in D. carota culture, showing the possible function of MATE involvement during anthocyanin accumulation to the tonoplast. Overall, the findings showed that salt stress stimulates the accumulation of anthocyanin in D. carota, which is mediated by changes in the transcription of key genes involved in their production and sequestration.

\section{ASSOCIATED CONTENT}

\section{SI Supporting Information}

The Supporting Information is available free of charge at https://pubs.acs.org/doi/10.1021/acsomega.1c02941.

Average expression stability values (M) of three candidate reference genes in $D$. carota cell culture growing in control and salt stress medium in the flask, average expression stability values (M) of three candidate reference genes in $D$. carota cell culture growing in control and salt stress medium in the bioreactor, list of candidate reference genes and target gene for $D$. carota cell culture used in a quantitative realtime polymerase chain reaction (qRT-PCR) analysis, $D$. carota reference genes in the flask at different time points ranked according to expression stability as determined by NormFinder and geNorm, D. carota reference genes in the bioreactor at different time points ranked according to expression stability as determined by NormFinder and geNorm, data used for plotting heatmap and volcano plot for flask, and data used for plotting heatmap and volcano plot for bioreactor (PDF)

\section{AUTHOR INFORMATION}

\section{Corresponding Author}

Nandini P. Shetty - Plant Cell Biotechnology Department, CSIR-Central Food Technological Research Institute, Mysuru
570020 Karnataka, India; 다이.org/0000-0002-86387511; Phone: 91-821-2516501; Email: nandinips@ cftri.res.in; Fax: 91-821-2517233

\section{Authors}

Kirti R. Saad - Plant Cell Biotechnology Department, CSIRCentral Food Technological Research Institute, Mysuru 570 020 Karnataka, India

Gyanendra Kumar - Plant Cell Biotechnology Department, CSIR-Central Food Technological Research Institute, Mysuru 570020 Karnataka, India

Sandeep N. Mudliar - Plant Cell Biotechnology Department, CSIR-Central Food Technological Research Institute, Mysuru 570020 Karnataka, India

Parvatam Giridhar - Plant Cell Biotechnology Department, CSIR-Central Food Technological Research Institute, Mysuru 570020 Karnataka, India; ○ orcid.org/0000-0001-72556268

Complete contact information is available at:

https://pubs.acs.org/10.1021/acsomega.1c02941

\section{Author Contributions}

The experiment was designed and supervised by N.P.S., S.N.M., and P.G. who also assisted in writing and editing the manuscript. K.R.S. and G.K. designed and performed the experiments and wrote the manuscript. Authors K.R.S. and G.K. equally contributed to this work and share equal authorship.

\section{Funding}

The authors are thankful to the CSIR-CFTRI for financial assistance. We are thankful to Director, CSIR-CFTRI, Mysore, for the research facilities. Authors K.R.S. and G.K. are grateful to CSIR, New Delhi, and ICMR, New Delhi, respectively, for the fellowship.

\section{Notes}

The authors declare no competing financial interest.

\section{ACKNOWLEDGMENTS}

This research was supported by the Central Food Technological Research Institute (CFTRI) funded by Council of Scientific and Industrial Research (CSIR). Authors K.R.S. and G.K. are grateful to CSIR, New Delhi and ICMR, New Delhi respectively for the fellowship. We would like to thank University of Mysore to help us for confocal microscopy pictures.

\section{ABBREVIATIONS}

$\mathrm{ACT}$, actin

$C L$, 4-coumarate: CoA-ligase

$\mathrm{C} 4 \mathrm{H}$, cinnamate-4-hydroxylase

$\mathrm{CHI}$, chalcone isomerase

CHS, chalcone synthase (CHS)

$\mathrm{CTAB}$, cetyl trimethylammonium bromide

DFR, dihydroflavonol 4-reductase

$\mathrm{F} 3 \mathrm{H}$, flavanone 3-hydroxylase

F3' $\mathrm{H}$, flavonoid $3^{\prime}$-hydroxylase

FW, fresh weight

GAPDH, glyceraldehyde-3-phosphate dehydrogenase

GI, growth index

IAA, indole acetic acid

Kin, kinetin

LDOX, leucoanthocyanidin dioxygenase 
MATE, multidrug and toxic compound extrusion

MS, Murashige and Skoog

$\mathrm{NH}_{4} \mathrm{NO}_{3}$, ammonium nitrate

$P A L$, phenylalanine ammonia lyase

$\mathrm{KNO}_{3}$, potassium nitrate

qRT-PCR, quantitative real time-polymerase chain reaction

TUB, tubulin

UFGT, UDP-glucose:flavonoid 3-O-glucosyltransferase

\section{REFERENCES}

(1) Khoo, H. E.; Azlan, A.; Tang, S. T.; Lim, S. M. Anthocyanidins and anthocyanins: colored pigments as food, pharmaceutical ingredients, and the potential health benefits. Food Nutr. Res. 2017, 61, 1361779.

(2) Vishnu, V. R.; Renjith, R. S.; Mukherjee, A.; Anil, S. R.; Sreekumar, J.; Jyothi, A. N. Comparative study on the chemical structure and in vitro antiproliferative activity of anthocyanins in purple root tubers and leaves of sweet potato (Ipomoea batatas). J. Agric. Food Chem. 2019, 67, 2467-2475.

(3) Goswami, G.; Nath, U. K.; Park, J.-I.; Hossain, M. R.; Biswas, M. K.; Kim, H. T.; Nou, I.-S. Transcriptional regulation of anthocyanin biosynthesis in a high-anthocyanin resynthesized Brassica napus cultivar. J. Biol. Res. 2018, 25, 19.

(4) Jiang, S.; Chen, M.; He, N.; Chen, X.; Wang, N.; Sun, Q.; Chen, $\mathrm{X}$. MdGSTF6, activated by MdMYB1, plays an essential role in anthocyanin accumulation in apple. Hortic. Res. 2019, 6, 40.

(5) Wang, J.; Hou, Q.; Li, P.; Yang, L.; Sun, X.; Benedito, V. A.; Wen, J.; Chen, B.; Mysore, K. S.; Zhao, J. Diverse functions of multidrug and toxin extrusion (MATE) transporters in citric acid efflux and metal homeostasis in Medicago truncatula. Plant J. 2017, 90, 79-95.

(6) Pérez-Díaz, R.; Ryngajllo, M.; Pérez-Díaz, J.; Peña-Cortés, H.; Casaretto, J. A.; González-Villanueva, E.; Ruiz-Lara, S. VvMATE1 and VvMATE2 encode putative proanthocyanidin transporters expressed during berry development in Vitis vinifera L. Plant Cell Rep. 2014, 33, $1147-1159$.

(7) Giri, C. C.; Zaheer, M. Chemical elicitors versus secondary metabolite production in vitro using plant cell, tissue and organ cultures: recent trends and a sky eye view appraisal. Plant Cell, Tissue Organ Cult. 2016, 126, 1-18.

(8) Jang, H.-R.; Lee, H.-J.; Shohael, A. M.; Park, B.-J.; Paek, K.-Y.; Park, S.-Y. Production of biomass and bioactive compounds from shoot cultures of Rosa rugosa using a bioreactor culture system. Hortic., Environ. Biotechnol. 2016, 57, 79-87.

(9) Thakore, D.; Srivastava, A. K.; Sinha, A. K. Mass production of Ajmalicine by bioreactor cultivation of hairy roots of Catharanthus roseus. Biochem. Eng. J. 2017, 119, 84-91.

(10) Zhong, J.-J.; Yoshida, M.; Fujiyama, K.; Seki, T.; Yoshida, T. Enhancement of anthocyanin production by Perilla frutescens cells in a stirred bioreactor with internal light irradiation. J. Ferment. Bioeng. 1993, 75, 299-303.

(11) Hiroyuki, H.; Kousuke, H.; Eiji, N.; Mariko, O.; Yoshihito, K.; Setsuro, H.; Takeshi, K. Enhanced anthocyanin production from grape callus in an air-lift type bioreactor using a viscous additivesupplemented medium. J. Biosci. Bioeng. 2002, 94, 135-139.

(12) Meyer, J. E.; Pépin, M.-F.; Smith, M. A. L. Anthocyanin production from Vaccinium pahalae: limitations of the physical microenvironment. J. Biotechnol. 2002, 93, 45-57.

(13) Deepthi, S.; Satheeshkumar, K. Cell line selection combined with jasmonic acid elicitation enhance camptothecin production in cell suspension cultures of Ophiorrhiza mungos L. Appl. Microbiol. Biotechnol. 2017, 101, 545-558.

(14) Isah, T. Stress and defense responses in plant secondary metabolites production. Biol. Res. 2019, 52, 39.

(15) Saad, K. R.; Parvatam, G.; Shetty, N. P. Medium composition potentially regulates the anthocyanin production from suspension culture of Daucus carota. 3 Biotech 2018, 8, 134.
(16) Saad, K. R.; Kumar, G.; Giridhar, P.; Shetty, N. P. Differential expression of anthocyanin biosynthesis genes in Daucus carota callus culture in response to ammonium and potassium nitrate ratio in the culture medium. 3 Biotech 2018, 8, 431.

(17) Chevala, N. P. K.; Chevala, N. T.; Dhanakodi, K.; Nadendla, R. R.; Nagarathna, C. K. In vitro accumulation of polyphenols in tea callus derived from anther. Pharmacogn. Mag. 2016, 12, S400.

(18) Ji, X.-H.; Wang, Y.-T.; Zhang, R.; Wu, S.-J.; An, M.-M.; Li, M.; Wang, C.-Z.; Chen, X.-L.; Zhang, Y.-M.; Chen, X.-S. Effect of auxin, cytokinin and nitrogen on anthocyanin biosynthesis in callus cultures of red-fleshed apple (Malus sieversii f. niedzwetzkyana). Plant Cell, Tissue Organ Cult. 2015, 120, 325-337.

(19) Chung, I. M.; Rekha, K.; Rajakumar, G.; Thiruvengadam, M. Elicitation of silver nanoparticles enhanced the secondary metabolites and pharmacological activities in cell suspension cultures of bitter gourd. 3 Biotech 2018, 8, 412.

(20) Anjum, S.; Abbasi, B. H.; Hano, C. Trends in accumulation of pharmacologically important antioxidant-secondary metabolites in callus cultures of Linum usitatissimum L. Plant Cell Tissue Organ Cult. 2017, 129, 73-87.

(21) Salmona, J.; Dussert, S.; Descroix, F.; De Kochko, A.; Bertrand, B.; Joët, T. Deciphering transcriptional networks that govern Coffea arabica seed development using combined cDNA array and real-time RT-PCR approaches. Plant Mol. Biol. 2008, 66, 105-124.

(22) Livak, K. J.; Schmittgen, T. D. Analysis of relative gene expression data using real-time quantitative PCR and the $2-\Delta \Delta \mathrm{CT}$ method. Methods 2001, 25, 402-408.

(23) De Spiegelaere, W.; Dern-Wieloch, J.; Weigel, R.; Schumacher, V.; Schorle, H.; Nettersheim, D.; Bergmann, M.; Brehm, R.; Kliesch, S.; Vandekerckhove, L.; Fink, C. Reference gene validation for RTqPCR, a note on different available software packages. PloS One 2015, 10, No. e0122515.

(24) Ram, M.; Prasad, K. V.; Singh, S. K.; Hada, B. S.; Kumar, S. Influence of salicylic acid and methyl jasmonate elicitation on anthocyanin production in callus cultures of Rosa hybrida L. Plant Cell Tissue Organ Cult. 2013, 113, 459-467.

(25) Maharik, N.; Elgengaihi, S. e.; Taha, H. Anthocyanin production in callus cultures of Crataegus sinaica boiss. Int. J. Acad. Res. 2009, 1, 30-34.

(26) Yin, Y.; Borges, G.; Sakuta, M.; Crozier, A.; Ashihara, H. Effect of phosphate deficiency on the content and biosynthesis of anthocyanins and the expression of related genes in suspensioncultured grape (Vitis sp.) cells. Plant Physiol. Biochem. 2012, 55, 7784.

(27) Ceoldo, S.; Levi, M.; Marconi, A. M.; Baldan, G.; Giarola, M.; Guzzo, F. Image analysis and in vivo imaging as tools for investigation of productivity dynamics in anthocyanin-producing cell cultures of Daucus carota. New Phytol. 2005, 166, 339-352.

(28) Kinnersley, A. M.; Dougall, D. K. Increase in anthocyanin yield from wild-carrot cell cultures by a selection system based on cellaggregate size. Planta 1980, 149, 200-204.

(29) Nagarajan, R. P.; Keshavarz, E.; Gerson, D. F. Optimization of anthocyanin yield in a mutated carrot cell line (Daucus carota) and its implications in large scale production. J. Ferment. Bioeng. 1989, 68, 102-106.

(30) Narayan, M.S.; Thimmaraju, R.; Bhagyalakshmi, N. Interplay of growth regulators during solid-state and liquid-state batch cultivation of anthocyanin producing cell line of Daucus carota. Process Biochem. 2005, 40, 351-358.

(31) Kobayashi, Y.; Akita, M.; Sakamoto, K.; Liu, H.; Shigeoka, T.; Koyano, T.; Kawamura, M.; Furuya, T. Large-scale production of anthocyanin by Aralia cordata cell suspension cultures. Appl. Microbiol. Biotechnol. 1993, 40, 215-218.

(32) Ochoa-Villarreal, M.; Howat, S.; Hong, S.; Jang, M. O.; Jin, Y.W.; Lee, E.-K.; Loake, G. J. Plant cell culture strategies for the production of natural products. BMB Rep. 2016, 49, 149.

(33) Jin, H.; Yu, Y.; Hu, S.; Quan, X.; Wu, S. Effect of nitrogen concentration, source, and phosphate concentration on accumulation of biomass and calycosin-7-O- $\beta$-D-glucoside in Astragalus membra- 
naceus adventitious roots. In Vitro Cell. Dev. Biol.: Plant 2020, 56, 407-414.

(34) Murthy, H. N.; Lee, E.-J.; Paek, K.-Y. Production of secondary metabolites from cell and organ cultures: strategies and approaches for biomass improvement and metabolite accumulation. Plant Cell, Tissue Organ Cult. 2014, 118, 1-16.

(35) Wu, C.-H.; Dewir, Y. H.; Hahn, E.-J.; Paek, K.-Y. Optimization of culturing conditions for the production of biomass and phenolics from adventitious roots of Echinacea angustifolia. J. Plant Biol. 2006, 49, 193-199.

(36) Patra, N.; Srivastava, A. K. Enhanced production of artemisinin by hairy root cultivation of Artemisia annua in a modified stirred tank reactor. Appl. Biochem. Biotechnol. 2014, 174, 2209-2222.

(37) Shinde, A. N.; Malpathak, N.; Fulzele, D. P. Studied enhancement strategies for phytoestrogens production in shake flasks by suspension culture of Psoralea corylifolia. Bioresour. Technol. 2009, 100, 1833-1839.

(38) Kokotkiewicz, A.; Luczkiewicz, M.; Kowalski, W.; Badura, A.; Piekus, N.; Bucinski, A. Isoflavone production in Cyclopia subternata Vogel (honeybush) suspension cultures grown in shake flasks and stirred-tank bioreactor. Appl. Microbiol. Biotechnol. 2013, 97, 84678477.

(39) Irshad, M.; Debnath, B.; Mitra, S.; Arafat, Y.; Li, M.; Sun, Y.; Qiu, D. Accumulation of anthocyanin in callus cultures of red-pod okra [Abelmoschus esculentus (L.) Hongjiao] in response to light and nitrogen levels. Plant Cell Tissue Organ Cult. 2018, 134, 29-39.

(40) Lulu, T.; Park, S.-Y.; Ibrahim, R.; Paek, K.-Y. Production of biomass and bioactive compounds from adventitious roots by optimization of culturing conditions of Eurycoma longifolia in balloon-type bubble bioreactor system. J. Biosci. Bioeng. 2015, 119, 712-717.

(41) Cui, X.-H.; Murthy, H. N.; Wu, C.-H.; Paek, K.-Y. Adventitious root suspension cultures of Hypericum perforatum: effect of nitrogen source on production of biomass and secondary metabolites. In Vitro Cell. Dev. Biol.: Plant 2010, 46, 437-444.

(42) Nguyen, D. Q.; Eamens, A. L.; Grof, C. P. L. Reference gene identification for reliable normalisation of quantitative RT-PCR data in Setaria viridis. Plant Methods 2018, 14, 24.

(43) Kumar, G.; Saad, K. R.; Puthusseri, B.; Arya, M.; Shetty, N. P.; Giridhar, P. Exogenous Serotonin and Melatonin Regulate Dietary Isoflavones Profoundly through Ethylene Biosynthesis in Soybean [Glycine max (L.) Merr.]. J. Agric. Food Chem. 2021, 69, 1888-1899.

(44) Hennayake, C. K.; Takagi, S.; Nishimura, K.; Kanechi, M.; Uno, Y.; Inagaki, N. Differential expression of anthocyanin biosynthesis genes in suspension culture cells of Rosa hybrida cv. Charleston. J. Plant Biotechnol. 2006, 23, 379-385.

(45) M'mbone, M. E.; Cheng, W.; Xu, L.; Wang, Y.; Karanja, B. K.; Zhu, X.; Liu, L. Identification and transcript analysis of MATE genes involved in anthocyanin transport in radish (Raphanus sativus L.). Sci. Hortic. 2018, 238, 195-203.

(46) Gomez, C.; Conejero, G.; Torregrosa, L.; Cheynier, V.; Terrier, N.; Ageorges, A. In vivo grapevine anthocyanin transport involves vesicle-mediated trafficking and the contribution of anthoMATE transporters and GST. Plant J. 2011, 67, 960-970.

(47) Gani, U.; Vishwakarma, R. A.; Misra, P. Membrane transporters: the key drivers of transport of secondary metabolites in plants. Plant Cell Rep. 2021, 40, 1-18. 